\title{
Eight-potential-well order-disorder ferroelectric model and effects of random fields
}

\author{
Zhi-Rong Liu and Bing-Lin Gu \\ Department of Physics, Tsinghua University, Beijing 100084, People's Republic of China \\ Xiao-Wen Zhang \\ State Key Laboratory of New Ceramics and Fine Processing, Department of Materials Science \\ and Engineering, \\ Tsinghua University, Beijing 100084, People's Republic of China
}

\begin{abstract}
An eight-potential-well order-disorder ferroelectric model was presented and the phase transition was studied under the mean-field approximation. It was shown that the two-body interactions are able to account for the first-order and the second order phase transitions. With increasing the random fields in the system, a first-order phase transition is transformed into a second-order phase transition, and furthermore, a second-order phase transition is inhibited. However, proper random fields can promote the spontaneous appearance of a first-order phase transition by increasing the overcooled temperature. The connections of the model with relaxors were discussed.
\end{abstract}

PACS:77.80.Bh 64.60.Cn 64.60.Kw 77.22.Ej

Typeset using REVTEX 
Relaxor ferroelectrics (relaxors) experience no macroscopic phase transition at zero electric field. 1 Nevertheless, a ferroelectric phase can be induced by an external electric field at low temperatures. For example, in $\mathrm{Pb}\left(\mathrm{Mg}_{1 / 3} \mathrm{Nb}_{2 / 3}\right) \mathrm{O}_{3}(\mathrm{PMN})$, a first-order induced phase transition, from the pseudo cubic phase to a macroscopically polar phase, has been disclosed by Ye et al. 2 A field-temperature phase diagram was proposed for PMN and a minimum threshold field strength $E_{t h}$ to induce the ferroelectric phase was determined 2 The random interactions between polar microregions and the random electric fields existing in the system were usually adopted to interpret the special characteristics of relaxors. 3 Glinchuk et al. showed that strong random fields can make the long-range order parameter disappear, and thus qualitatively explained the absence of the zero-field phase transition in relaxors.6 However, they only considered a second-order phase transition and no external electric field was involved. It appears, therefore, necessary to investigate the effects of random fields and external fields on the first-order phase transition.

A pseudospin model is usually used to interpret the phase-transition phenomena of order-disorder ferroelectrics in which the active ions have two equilibrium sites. In order to explain the first-order phase transition, four-spin coupling terms should be introduced and the four-body coupling strength is required to be larger than the two-body one. $\mathrm{G}$ On the other hand, PMN presents a disordered pseudo cubic structure with important atomic shifts around the special positions of the ideal perovskite structure, ${ }^{9}$ i.e., the polar ions in PMN have more than two equilibrium positions. Therefore, a multi-direction "spin" system should be studied in order to understand the special properties of PMN.

For the reasons mentioned above, we present an eight-potential-well ferroelectric model and investigate the the phase transition under various condictions in this paper.

For PMN, the electric field-induced macropolar phase has trigonal symmetry with point group $3 m, 2$ which suggests that for each polar ion there are eight potential wells along $\{111\}$ equivalent directions. When an external field is applied along one of eight $\{111\}$-equivalent directions, the Hamiltonian matrix of the order-disorder system with eight potential wells is assumed to be: 


$$
H=\left[\begin{array}{cccccccc}
-E p_{0} & \frac{\Omega}{2} & 0 & 0 & 0 & 0 & 0 & 0 \\
\frac{\Omega}{2} & E p_{0} & 0 & 0 & 0 & 0 & 0 & 0 \\
0 & 0 & -\frac{1}{3} E p_{0} & \frac{\Omega}{2} & 0 & 0 & 0 & 0 \\
0 & 0 & \frac{\Omega}{2} & \frac{1}{3} E p_{0} & 0 & 0 & 0 & 0 \\
0 & 0 & 0 & 0 & -\frac{1}{3} E p_{0} & \frac{\Omega}{2} & 0 & 0 \\
0 & 0 & 0 & 0 & \frac{\Omega}{2} & \frac{1}{3} E p_{0} & 0 & 0 \\
0 & 0 & 0 & 0 & 0 & 0 & -\frac{1}{3} E p_{0} & \frac{\Omega}{2} \\
0 & 0 & 0 & 0 & 0 & 0 & \frac{\Omega}{2} & \frac{1}{3} E p_{0}
\end{array}\right],
$$

where $E$ is the electric field strength, and $p_{0}$ is the magnitude of the dipole when an ion locates in a certain well. A factor $1 / 3$ is introduced in terms of Eq. (1) when the directions of the well are not parallel to that of the external field. $\Omega$ is the tunneling frequency. Only the tunneling between wells with opposite directions are considered here. In ferroelectric systems, polar ions interact with each other and with different kinds of defects in the system. Under a mean field approximation, the interactions upon a certain ion may be represented by an equivalent field, i.e.,

$$
E p_{0}=J \frac{\langle p\rangle}{p_{0}}+E_{\text {ext }} p_{0}+E_{\text {rand }} p_{0},
$$

where $\langle p\rangle$ is the thermal average value of dipole moments, and $J$ is the coupling energy. Only two-body interactions are involved here because a linear relation is assumed between $\langle p\rangle$ and $E . E_{\text {ext }}$ is the applied external electric field. $E_{\text {rand }}$ is the internal random fields in the system which come from the direct interactions of dipoles, fields created by point charge defects and atomic ordering, etc.. $E_{\text {rand }}$ is assumed to have a Gaussian distribution:

$$
\rho\left(E_{\text {rand }}\right)=\frac{1}{\sqrt{2 \pi} \sigma_{e}} \exp \left[\frac{-E_{\text {rand }}^{2}}{2 \sigma_{e}^{2}}\right],
$$

where $\sigma_{e}$ is the distribution width. By using Eqns. (1)-(3), the order parameter $\langle p\rangle$ can be determined for any temperature.

At first, the phase transition temperatures are calculated when there is no external field and random fields, which are shown in Fig. 1. It can be seen that the long-range order 
can appear only at $\Omega \leq 2 J$. If the tunneling movement does not exist $(\Omega=0)$, the critical temperature is $T_{c}=\frac{J}{3 k_{B}}$ where $k_{B}$ is the Boltzmann constant. An interesting characteristic of Fig. 1 is that both the second-order phase transition and the first-order phase transition exist for the present model. It is quite different from the case of two-direction pseudospin model where no first-order phase transition exists if the number of coupled spins is two. In Fig.1, there is a tricritical point between the second- and the first-order phase transitions, which is determined as $\Omega_{t r i}=0.56 \mathrm{~J}$ and $T_{t r i}=0.23 \mathrm{~J} / k_{B}$. For a first-order phase transition, the overcooled temperature $T_{-}$decreases rapidly with increasing $\Omega$ and vanishes at $\Omega=\frac{2}{3} J$. When $\frac{2}{3} J<\Omega<2 J$, the long-range order cannot spontaneously appear in a cooling process, while a polar state can be induced at low temperatures. This is qualitatively consistent with the behavior of PMN 目

Solid lines in Fig. 2 depict the phase transition temperatures versus external field when $\Omega=J$ and $\sigma_{e}=0$. At small external fields, the phase transition is the first-order type. And at large external fields, a second-order phase transition occurs. A tricritical point also appears here. For a first-order phase transition, the induced ferroelectric phase disappeares at the overheated temperature in a heating process. It is seen from the figure that the overheated temperature increases with increasing the field strength, whereas the zero-field depoling of an induced state occurs always at the temperature $T_{d o}$ (see Fig. 2), independently of the initial poling field. The existence of $T_{d o}$ and the tricritical point agrees with the experiments in PMN.210 The curve of the overcooled temperature can also be interpreted as the curve of the threshold field strength $E_{t h}$ to induce a ferroelectric state at different temperatures. $E_{t h}$ decreases with decreasing temperature in our calculation. In experiments,2 however, $E_{t h}$ decreases firstly and then increases with decreasing temperature. The discrepancies come from the dynamical process, which is not considered in the calculation. At low temperatures, the system is frozen into a glass-like state. so slow that the induced polar phase cannot come into being even if the polar phase has smaller free energy. As a result, $E_{t h}$ increases at low temperatures $\left(T<T_{d o}\right)$.

In Fig. 2, when the external field is larger than the tricritical point, there is no "real" 
phase transition in the sense that the spontaneous breakdown of symmetry does not occur. Nevertheless, the curves of the average polarization $\langle p\rangle$ in cooling processes (see Fig. 3) indicate that $\langle p\rangle$ increases rapidly in a small temperature range but changes slowly at other temperatures when the external field is a little larger than the tricritical value (curve $\mathrm{c}$ in Fig. 3). Thus, a second-order "phase transition" may be approximately defined at the temperature where $\langle p\rangle$ changes most quickly. When the external field is much larger than the tricritical value (curve d in Fig. 3), $\langle p\rangle$ varies rather smoothly, and the phase transition is difficult to determine. The features of the polarization curves in Fig. 3 are consistent with experiments.11

To explore the effects of random fields, the phase transition temperatures for the random fields $\sigma_{e}=0.2 \mathrm{~J} / p_{0}$ are also depicted in Fig.2 (dashed lines). Compared with the curves with no random field (solid lines), the critical temperature and the overheated temperature decrease, and the range of the first-order phase transition gets smaller, i.e., the phase transition is compressed. It should be noted that the overcooled temperature increases when random fields exist. Thus the phase transition is promoted by the random fields in the sense that a spontaneous phase transition is easier to occur, which is in conflict with the common sense.

The continuous effects of random fields on the phase transition temperatures are shown in Fig. 4 for $\Omega=J$. By enhancing random fields, the interval between the overheated and the overcooled temperatures shrinks gradually, and vanishes at a tricritical point. Beyond the tricritical point, the random fields make the critical temperature decrease, and finally inhibit the phase transition completely. The promotion effect of random fields on the spontaneous first-order phase transition is obvious in Fig. 4: the critical temperature keeps approximately constant while the overcooled temperature increases extraordinarily. This unusual effect seems to challenge the previous idea that the phase transition in PMN is inhibited by strong random fields. Perhaps the actual situation is that the random fields are not strong enough to promote a spontaneous phase transition.

At last, a phase diagram on the $\Omega-\sigma_{e}$ plane is given in Fig. 5. The $\Omega-\sigma_{e}$ plane is divided into three regions where a first-order phase transition, a second-order phase transition and 
no phase transition can occur, respectively. The boundary line between the first-order and the second-order phase transition regions is a tricritical line that is composed of tricritical points.

In summary, the phase transition of an eight-potential-well order-disorder ferroelectric system was investigated in this paper. The effects of the couplings, the external fields, and the internal random fields are discussed. The connections of the model with PMN were discussed.

This work was supported by the Chinese National Science Foundation and State Key Program of Basic Research Development, and the authors would like to thank Dr. Jian-She Liu for helpful discussions. 


\section{REFERENCES}

${ }^{1}$ L. E. Cross, Ferroelectrics 76, 241 (1987).

2 Z. G. Ye and H. Schmid, Ferroelectrics 145, 83 (1993).

${ }^{3}$ D. Viehland, S. J. Jang, and L. E. Cross, J. Appl. Phys. 68, 2916(1990).

${ }^{4}$ H. Gui, B. L. Gu, and X. W. Zhang, Phys. Rev. B 52, 3135 (1995).

${ }^{5}$ V. Westphal, W. Kleemann, and M. D. Glinchuk, Phys. Rev. Lett. 68, 847 (1992).

${ }^{6}$ M. D. Glinchuk and V. A. Stephanovich, J. Phys.: Condens. Matter 6, 6317 (1994).

${ }^{7}$ R. Blinc and B.Zeks, Soft Modes in Ferroelectrics and Antiferroelectrics (North-Hollad, Amsterdam, 1974).

${ }^{8}$ C. L. Wang, Z. K. Qin, and D. L. Lin, Phys. Rev. B 40, 680 (1989).

${ }^{9}$ N. de Mathan, E. Husson, G. Calvarin, J. R. Gavarri, A. W. Hewat, and A. Morell, J. Phys.: Condens. Matter 3, 8159 (1991).

${ }^{10}$ G. Calvarin E. Husson, and Z. G. Ye, Ferroelectrics 165, 349 (1995).

11 Z. G. Ye, Key Engineering Materials 155-156, 81 (1998). 


\section{FIGURES}

FIG. 1. Phase transition temperatures as functions of tunneling frequency with no random fields. The solid line, the dashed line, and the dotted line represent the critical, the overheated, and the overcooled temperatures, respectively. The temperatures and the tunneling frequency are measured in units of $J / k_{B}$ and $J$ respectively.

FIG. 2. Phase transition temperatures as functions of external field when $\Omega=J$. The lines above tricritical points represent critical temperatures. Below tricritical points, lines on the left represent overcooled temperatures, and that on the right represent overheated temperatures. The solid and dashed lines correspond to the case of $\sigma_{e}=0$ and $0.2 J / p_{0}$, respectively. External fields are measured in unit of $J / p_{0}$ and temperatures in unit of $J / k_{B}$.

FIG. 3. Average polarization $\langle p\rangle$ (in unit of $p_{0}$ ) as function of temperature (in unit of $J / k_{B}$ ) in cooling processes at $\Omega=J$ and $\sigma_{e}=0$. Curves a, b, c, and d correspond to the external field $E_{\text {ext }}=0.075,0.095,0.115$, and $0.15 \mathrm{~J} / p_{0}$, respectively.

FIG. 4. Phase transition temperatures (in unit of $J / k_{B}$ ) versus random fields width (in unit of $\left.J / p_{0}\right)$ when $\Omega=J$. The solid, dashed and dotted lines represent the critical, overheated, and overcooled temperatures, respectively.

FIG. 5. Phase diagram on the $\Omega-\sigma_{e}$ plane. $\alpha, \beta$, and $\gamma$ designate the regions where the first-order, the second-order, and no phase transition can occur. $\Omega$ and $\sigma_{e}$ are measured in units of $J$ and $J / p_{0}$, respectively. 


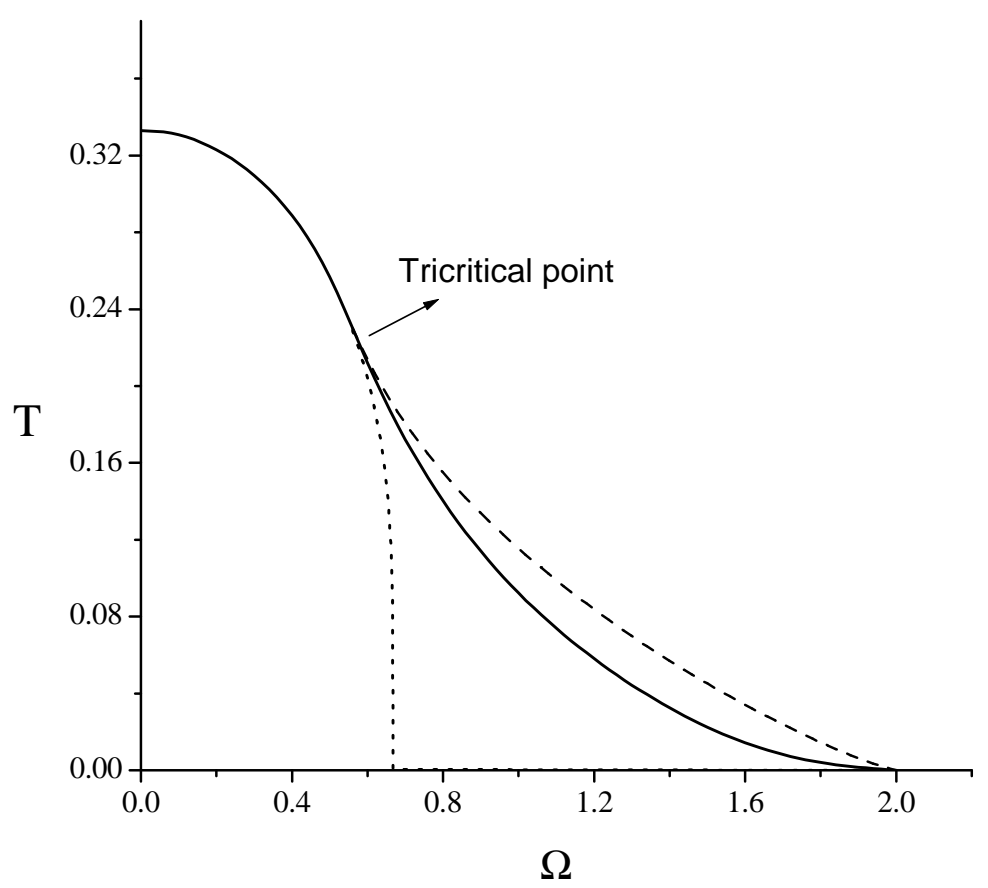




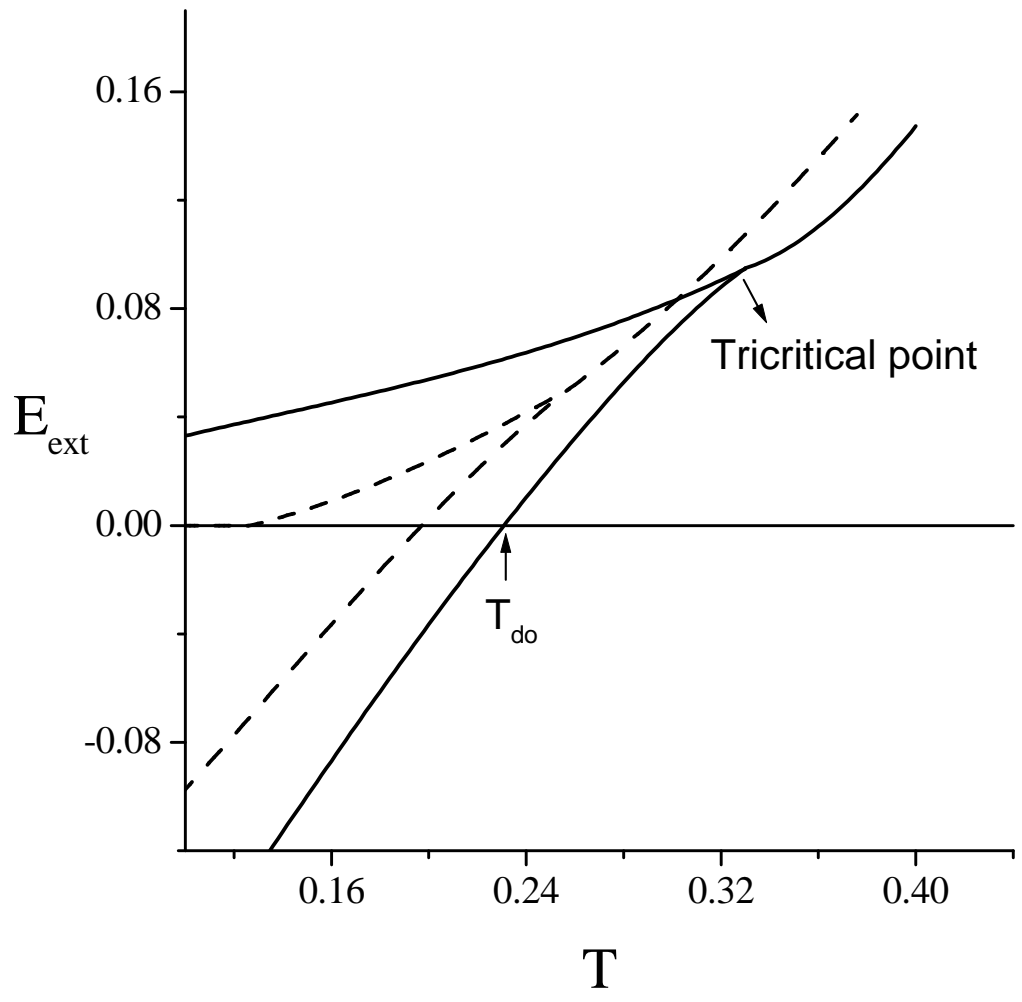




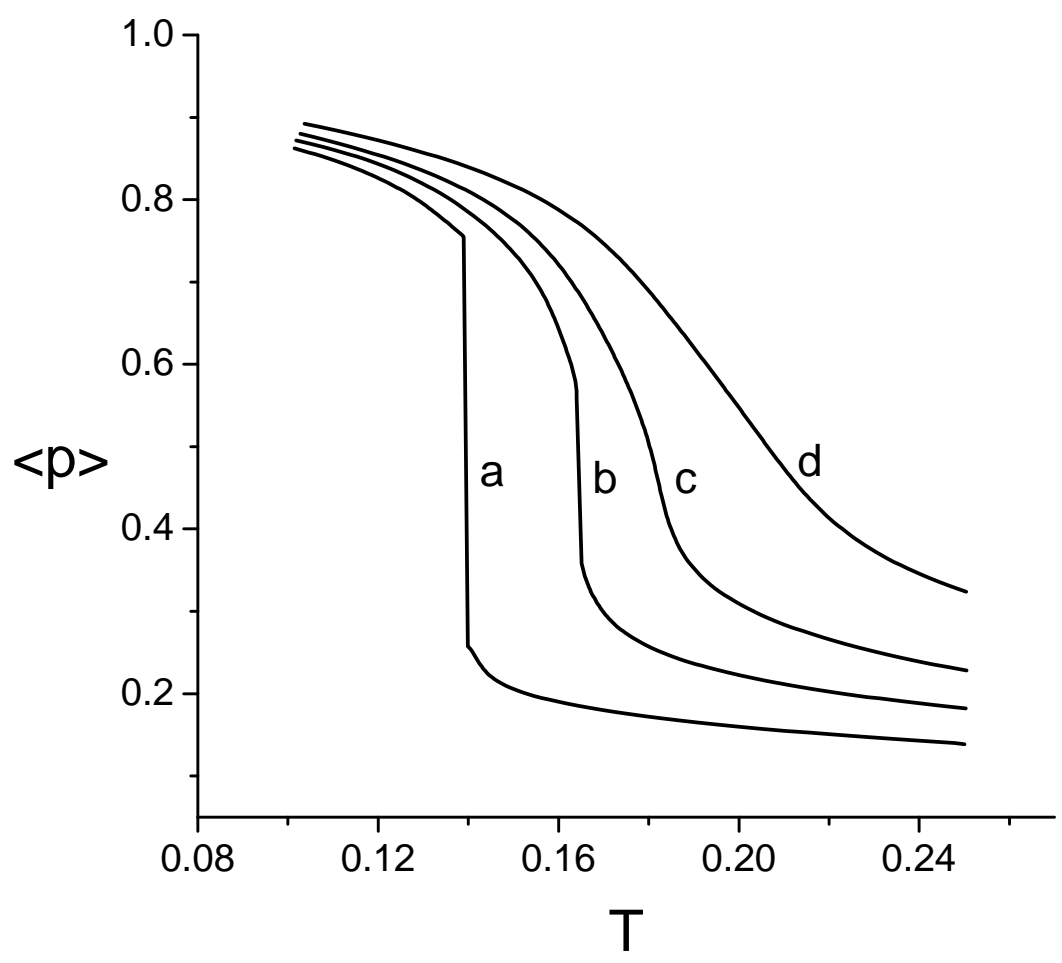




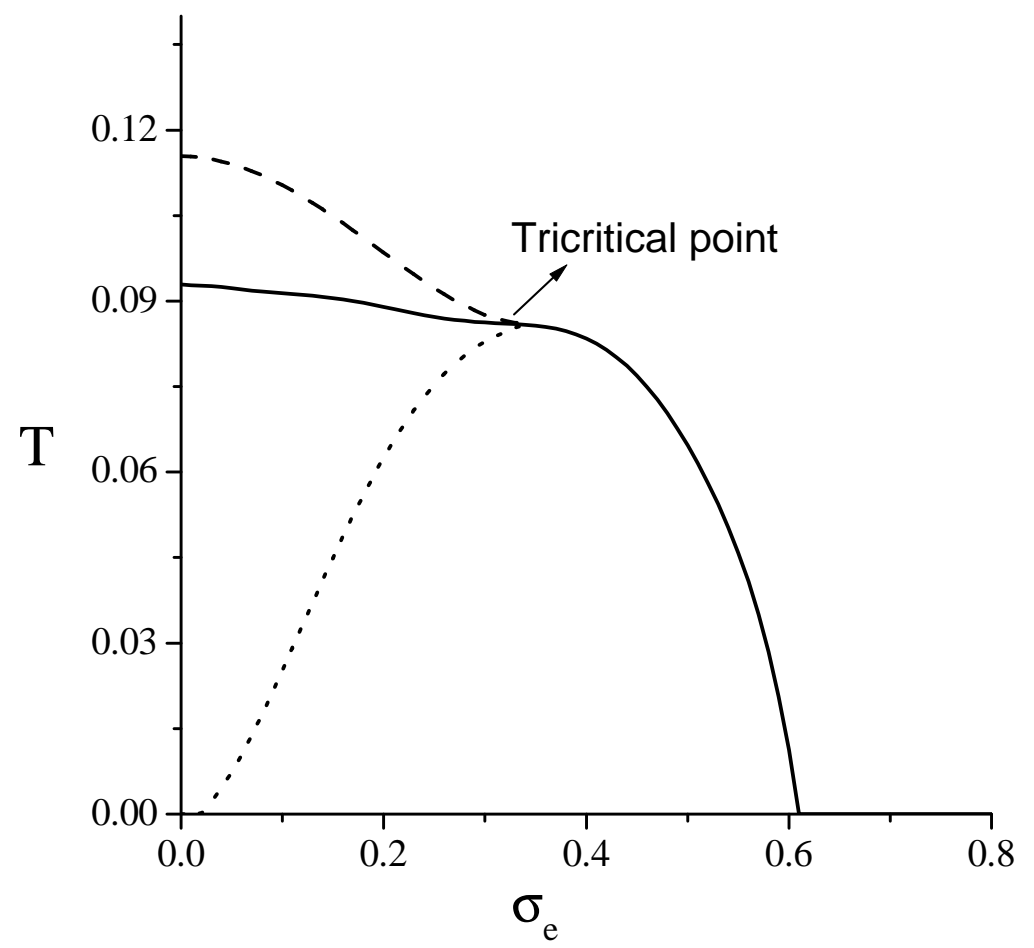




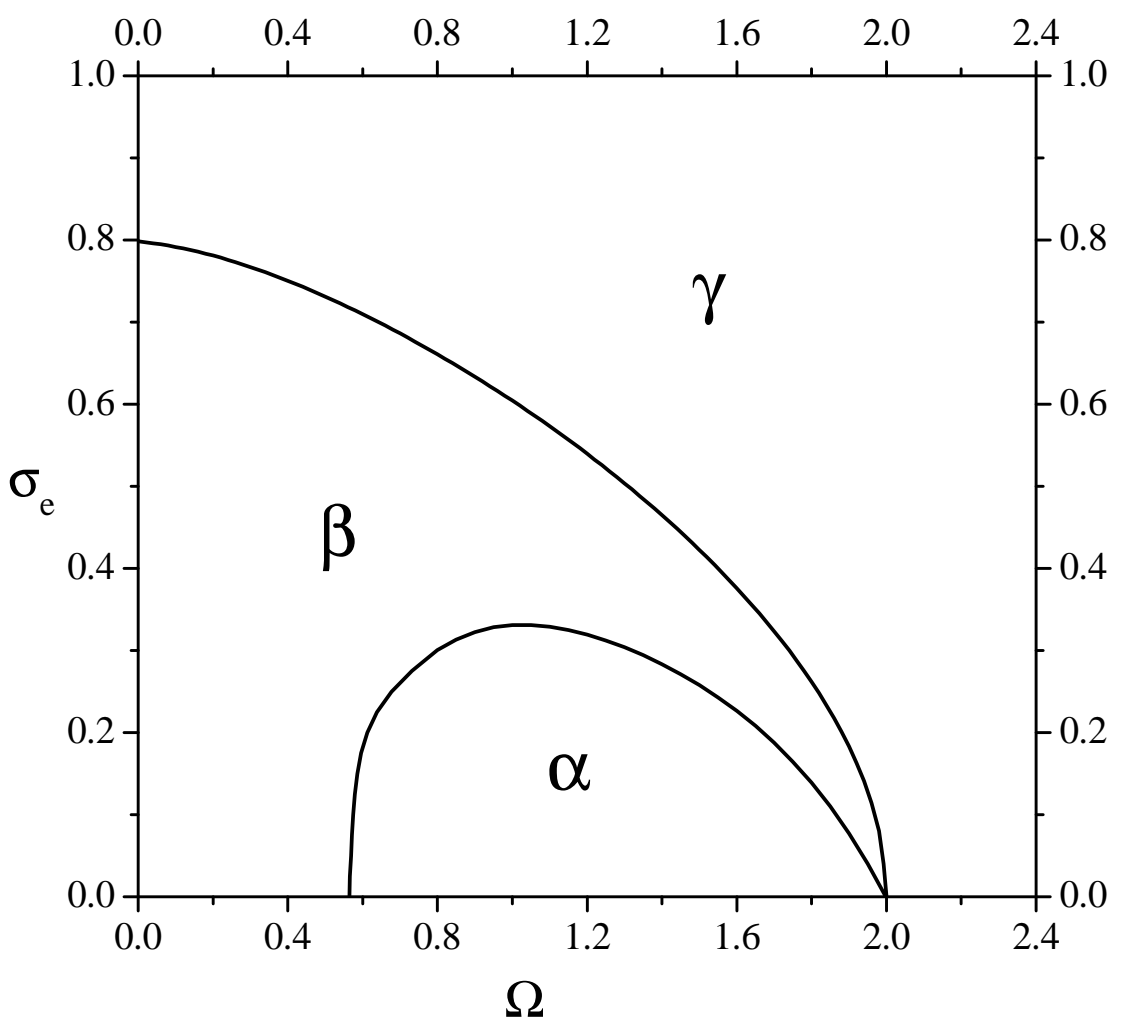

